\title{
Novel synthesis of nanoparticles from cockle shells via mechanical method for cytarabine drug release
}

\begin{abstract}
The current mechanical method of modification resulted in producing nanoparticle powders used as the biologically active material. The given method was employed to improve the CCANPs properties making the particles proper in the field of medicine. This study was synthesis nanoparticles from cockle shall, and it analyses the phase purity of the mineral and physicochemical composition of Cockle Shall, and it also analyses the crystallographic structures of the powders. Cytarabine (Ara-C) drug is used to treat acute myeloid leukaemia, and this is due to it's very low biological halflife; it must frequently be used so as to treat leukaemia by attacking all the divided cells cancer and healthy cells. The process involves aims to protect Cytarabine from fast elimination and degradation that was caused by the encapsulation of drug in pharmaceutically promising carriers. The calcium carbonate aragonite nanoparticles (CCANPs) was formulated for improving the therapeutic efficacy by targeting the tumorous site dependent on $\mathrm{pH}$. The Ara-C was loaded in CCANPs; it was found that Ara-C was relatively more stable at normal $\mathrm{pH}$ (7.4), and this resulted in decreasing the release of Ara-C, but the CCANPs was gradually dissociated in acidic $\mathrm{pH}$ (4.8) regimes which triggered the faster release of Ara-C. The CCANPs was characterised before and after the loaded Ara-C to determine the particle size and morphological properties using TEM, FESEM. The physiochemical features were detected using XRD, FTIR, Zeta potential, drug loaded and drug release. The findings of the proposed method to synthesise the nanoparticles improved the performance of CCANPs. The findings showed that the particles shape of CCANPs was spherical and ranged 20-50 nm without marking differences after loading the Ara-C. Moreover, FTIR results confirmed that there are no noticeable changes before and after the loading of Ara-C which refers to the encapsulation process which positively reflects on the Ara-C activity.
\end{abstract}

Keyword: $\mathrm{CACO} 3$ aragonite nanoparticles; Cockle shall; Cytarabine; Particle size; Physicochemical properties 